\title{
SOFT X-RAY CAMERA FOR INTERNAL SHAPE AND CURRENT DENSITY MEASUREMENTS ON A NONCIRCULAR TOKAMAK
}

\author{
R.J. Fonck, K.P. Jaehnig, E.T. Powell, M. Reusch, P. Roney, : רd M. P. Simon
}

\author{
Princeton Plasma Physics Laboratory \\ Princeton, New Jersey 08543 USA
}

PPPL- 2518

JE88 011872

\begin{abstract}
Soft X-ray measurements of the intemal plasma flux surface shapes in pr. 1ciple allow a determination of the plastna current density distribution, and provide a neck . ary monitor of the degree of internal elongation of tokamak plasmas with a noncircular cross section. A two-dimensional, tangentially viewing, soft $\mathrm{X}$-ray pinhole camera has been fabricated to provide internal shape measurements on the PBX-M tokamak. It consists of a scintillator at the focal plane of a foil-filtered pinhole camera, which is, in turn, fiber optically coupled to an intensified framing video camera ( $\Delta t \geqslant 3 \mathrm{msec}$ ). Automared data acquisition is performed on a stard-alone image-processing system, and data archiving and retrieval takes place on an optical disk video recorrm. The entire diagnostic is controlled via a PDP-11/73 microcomputer. The derivation of the poloidal emission distribution from the measured image is done by fiting to model profiles.
\end{abstract}

\section{DISCLAIMER}

This repart was prepared as an account of wort sponsored by ap agency of the United States Government. Neither the United States Government nor any ayency thereuf. nor any of their employees, makes any warraniy, express of implied, or essumes any legal liability of responsibility for the accuracy, compleieness, or usefulness of any information, apparatus, product, or process disclosed, or represents that its use would not infrioge privately owned rights. Referprocess disclosed, or represents that its use would not infrein to any specific commercial product, process, of service by trade name, tiademark, manufacturer, or otherwise does not necessarily constitute of imply its endorsement, recommendation, of favoring by the United States Government or any agency thereul. The views and opinions of authors expressed hercin do not necessarily state or reflect those of the United States Government or any agency thereof.

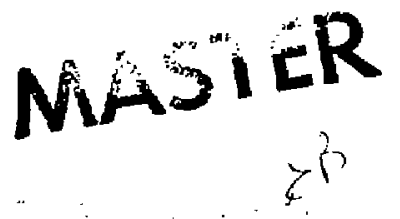




\section{Introduction}

The ability to measure the internal shape of the magnetic flux surfaces in a noncircular tokamak plasma provides a means of gaining significant information on the nature of the plasma equilibrium and possibly allows a measurement of the plasma current density profile, $j(R)$. In fact, a sufficiently accurate measurement of the intemal plasma flux surface shapes allows a direct determination of the plasma cursent distribution, ${ }^{1}$ but uncertainties in the measurements may make such a derivation problematic. However, a combination of external magnetics measurements plus a knowledge of the internal shape should at least allow a determination of the central rotational transform, $q(0)$, via magnetic equilibrium calculations. In the present and next generation of highly noncircular advanced tokamak experiments, a knowledge of $j(R)$ or $q(0)$ is crucial for understanding the plasma stability at high beta and as a means of monitoring efforts to control actively the plasma current profiles. For these reasons, we have started the development of a simple diagnostic system to determine accurately and routinely the internal shape of tokamak plasmas.

A common technique for the measurement of the internal plasma cross section in tokamaks has been the measurement of the spatial distribution of soft X-ray (SXR) emissions (1-10 keV) from the hot plasma core. ${ }^{2}$ Arising from continuum and metallic lines emission, the equal emissivity contours of this radiation are tightly coupled to isothermal surfaces in the plasma. Assuming the plasma flux surfaces have a constant temperature, determination of the isotherms then gives the internal flux surface shape. Two basic approaches have been used in the past for such SXR measurements. In the first, tomographic inversions of multi-chordal views of the plasma from several one-dimensional $\mathrm{X}$-ray diode cameras are used to provide a map of the poloidal cross section, $\mathbf{3 , 4}$ while the second technique uses a single tangentially viewing SXR two-dimensional camera to measure the plasma cross section. 5.6 While both approaches have their advantages, recent developments in the PBX-M tokamak group have pointed out their complementary nature. In particular, Holland et al. ${ }^{7}$ point out that the use of a low-time resolution image from a tangentially viewing camera allows a reasonable reconstruction of high frequency local perturbations in the poloidal cross section observed with a minimal set of high-time resolution surface-barrier or PIN diode array cameras. This combined use of a slow SXR tangential camera plus a minimal set of one-dimensional $\mathbf{X X}$ diode array cameras viewing the poloidal cross section is especially important for the diagnosis of tokamaks with limited 
physical access, such as the planned CIT facility.

In an earlier paper, we described a prototype soft $X$-ray pinhole camera system which was developed for internal shape measurements on the noncircular PBX tokamak plasmas. ${ }^{6}$ Here, we describe a new design of a soft $X$-ray pinhole camera diagnostic developed for the PBX-M experiment, with an emphasis on changes or improvements with respect to the prototype camera and data acquisition system described earlier. The emphasis in the development of this system has been to produce an economical means of providing routine measurements of the internal plasma shape. Ease of use, minimal required access to the vacuum vessel, passive measurement, and insensitivity to magnetic field and plasma density were all considerations in the design of this system. Rapid access to, analysis of, and display of data were also prime requirements of the system design.

PBX-M itself is a moderate-sized tokamak experiment with an outer cross-sectional shape resembling that of a kidney bean, with the indentation on the inboard side of the plasma major radius. The plasma has a major radius of $R=1.65 \mathrm{~m}$, a minor radius of $a=$ $0.30 \mathrm{~m}$ at the midplane waist of the bean-shaped cross section, and a vertical elongation of about 2 . Initial estimates of the expected plasma equilibria and shapes in PBX-M indicate that the vertical elongation of the flux surface at $a / 2$ is roughly proportional to $q(0)$, and an accuracy of $10 \%$ or better in the measured shapes is needed to infer $\mathrm{q}(0)$ to the desired accuracy of $10 \%$.

\section{Apparatus}

As described in our earlier paper, we use a tangentially viewing two-dimensional pinhole camera situated in the torus midplane to record simultaneously the entire plasma cross section. Assuming toroidal symmetry or, at least, a known toroidal dependence in the SXR emissivity, an inversion algorithm can be used to derive the poloidal cross section of the emissivity contours from the tangential chordally integrared image.6,8 The desired time resolution of the camera is typically on the order of $5 \mathrm{msec} / \mathrm{frame}$, which is shorter than the large macroscopic changes in the plasma parameters, but still long enough to average over high frequency (5-100 kHz) local perturbations in the plasma interior.

A schematic of the camera optical train is shown in Fig. 1. A $400 \mu \mathrm{m}$ diameter pinhole drilled in a stainless steel block defines the imaging element. The optical axis of the camera 
views the plasma with a tangency radius of $145 \mathrm{~cm}$ and an angular field of view of $\pm 30^{\circ}$ in both the horizontal and vertical directions. The camera pinhole is located at a radius of 213 $\mathrm{cm}$ while the plasma extends from $100-195 \mathrm{~cm}$ horizontally and $\pm 70 \mathrm{~cm}$ vertically. A remotely actuated filter selector allows a variety of SXR or vacuum ultraviolet filters to be placed in front of the pinhole. Typically, a thin Be foil filter is used to define the low energy ( $1 \mathrm{keV}$ ) sensitivity cut-off of the camera, while the rapid drop in SXR intensity from the plasma and the high-energy insensitivity of the thin image converting surface result in a high energy cut-off of the camera sensitivity approximately in the $10-20 \mathrm{keV}$ photon energy range.

An X-ray to visible light converter is coated on the surface of a vacuum-sealed fiber optic faceplate to produce a visible optical image which is then transmitted outside the magnetic field region through a coherent optical fiber image conduit to an intensified fast scanning two-dimensional photodiode array. In the present system, a thin coating of P-20 phosphor is used as the SXR-to-visible converter. This phosphor, which consists of a polycrystalline silver-activated zinc-cadmium-sulfide powder, is similar to zinc-sulfide, which is known to have a very high scintillation efficiency. 9 While it is limited to thin screens due to its opacity to its own luminescence, it does appear to be quite serviceable for our low photon energy range (i.e., $\sim 0.1-10 \mathrm{keV}$ ). Laboratory tests with an electron-beam SXR source showed this to be a reasonably efficient converter medium with the requisite spatial resolution needed for the compact camera head at the vacuum vessel. Further tests are presently underway to determine more accurately the exact energy sensitivity of this image converter.

In the first version of the camera optics, the single-stage microchannel plate (MCP) image intensifier is lens-coupled to the image conduit, and visible light tests showed the detector system to be sufficiently efficient to avoid MCP saturation at integration times down to 5 msec. The system is presently being rebuilt to provide better efficiency through fiber optic coupling directly to the fiber optic input window of a new image intensifier, which has a P-20 output phosphor instead of the P-11 used in the present intensifier. This will provide a more favorable spectral distribution of the intensified image for matching the sensitivity of the photodiode array . Earlier tests of these P-20 phosphor screens showed that the decay time of the phosphor is typically $\sim 1$ msec, 10 which is sufficient for our goal of 3-5 msec resolution. 
The detector is a $128 \times 128$ pixel self-scanned photodiode array with a $60 \mu \mathrm{m}$ center-tocenter pixel spacing (Reticon Model MC9128). This detector has the advantages of ready commercial availability, good dynamic range $(1000 ; 1)$, and fast readout time $(\geq 3$ $\mathrm{msec}$ /frame). It does exhibit a slight degree of nonlinearity in its output as a function of total input energy, which is readily calibrated with a constant source. The synchronization and readout electronics for the camera head are contained in the Reticon Model MB9000 Video Formatter system available with the camera head. The camera outputs a nonstandard video sigral, consisting of an analog video signal accompanied by digital timing pulses for the master pixel clock, the line enable , and total frame enable signals.

Given the potentially large amount of data obtained with a video system $(\sim 0.5-4$ $\mathrm{MB}$ /discharge), and the need to retrieve and analyze rapidly large amounts of data from the archival system, we designed a stand-alone data acquisition and archival system to support this pinhole camera diagnostic. The use of such a stand-alone system puts minimal demand on the tokamak diagnostic acquisition computer system and allows fast transfer of large amounts of video data. A schematic of the system electronics and acquisition apparatus is shown in Fig. 2. All communications between the apparatus in the tokamak test cell and the local data acquisition system are done through analog and digital fiber optically coupled data links.

In addition to the camera itself, the acquisition and archival system consists of three major components. First, a real-time image processing system from Recognition Concepts, Inc. (RCI TRAPIX Model 55/256) provides an 8-bit, $8 \mathrm{MHz}$ digitizer to synchronize with and digitize the incoming nonstandard video signal, and then store it in its local image memory (total of $8 \mathrm{MB}$ ) as a series of $16128 \times 128$ fields in each of its standard $512 \times 512$ 8-bit video image memory frames. In practice, only 0.5-2 MB of image memory is used for actual data acquisition, with the rest employed for local temporary storage and image processing. This results in 32 to 128 images acquired per discharge. The TRAPLX is a highly versatile platform for image acquisition and analysis. It can readily acquire image data from both the nonstandard video source from our camera and from standard RS-170 or NTSC video signals. It also provides the capability of high speed histograms, pseudo-color outpui, and a high-speed pipeline processor plus standard look-up tables for image processing functions such as dark field subtractions, gain corrections, and spatial smoothing. The RGB output of the TRAPLX is in standard RS170 video format. In addition to providing a high speed digitizer and buffered memory for 
data acquisition, this real-time image processor allows very rapid and efficient manipulation and analysis of the image data.

The second major data acquisition system component is the video optical disk recorder (ODR) (Panasonic Model TQ-2026F), which is used for primary data archiving in an analog video format. This approach was chosen for its economy, speed, and flexibility, Since the original data are digitized to 8-bit depth, standard video quality components can be used without loss of dynamic range. Also, all data transfer is done through video signals (i.e., $30 \mathrm{frames} / \mathrm{sec}$ ), allowing very fast archiving and access. Each disk holds 24,000 frames with a maximum access time of $0.5 \mathrm{sec}$ for any frame. A $128 \times 128$ data field in the TRAPLX image memory is zoomed up to a $256 \times 256$ image before writing to the ODR to avoid loss of spatial resolution of the data. Combined with timing labels and a reference intensity scale, this results in storing two time-exposures per video disk frame, resulting in an equivalent storage of $-375 \mathrm{MB}$ per disk, enough for about 1 week of tokamak operation. Retrieval of data from the ODR is accomplished by digitizing the standard NTSC video signal from the desired frame back into the TRAPIX. After a $2 \times 2$ convolution and reduction by 2 in size, the resulting recovered data have a net system noise of 1 bit or less. A further advantage of the chosen ODR is its internal micro-processor control, which allows easy access and control via an ASCII interface to a terminal or computer and provides the capability for a programmed loop playback of a data sequence: while the TRAPIX is used for quantitative image analysis.

The third major component is the local DEC PDP- $11 / 73$ microcomputer which provides overall system control and communication. The TRAPLX interface is connected directy to the computer Q-bus, and can provide direct access to image memory. Control and programming of the optical disk recorder is performed through one of the standard RS-232 ports. Likewise, a relatively slow ( 9600 baud) RS-232 link allows communication and some data transfer to the laboratory VAX cluster. Real-time synchronization of the system to the plasma shot is suppored by proyiding an interrupt on a repeatedly polled RS-232 serial line at the same time the camera readout is halted, and the TRAPIX is initialized for data acquisition. Camera readout is then restarted by a trigger synchronized with the plasma discharge time.

Mechanical control of the filter wheel stepper motor, control of the high voltage power supplies, and remote access to the camera readout control for variation of integration times 
are enacted via the facility-wide diagnostic data acquisition and control CAMAC highway.

\section{Performance and Sample Results}

The first generation of this SXR pinhole camera has operated on PBX-M since early 1988, and has allowed tests of most of the major components, including the basic camera design and the ODR-based archival system. We have not yet installed the variable filter drive or the CAMAC-based readout control electronics, so that changes in the integration time can be made only through access to the tokamak experimental cell. The fixed Befoil thickness is $25 \mu \mathrm{m}$. To date, SXR images of PBX-M plasmas have been obtained for a range of discharges with ohmic and neutral beam heating.

In general, the system is sensitive enough to allow routine measurements of ohmic plasmas with an integration time of $\Delta t=20 \mathrm{msec}$ and $\Delta t=5-10 \mathrm{msec}$ for the hotter beamheated plasmas. With the installation of the fiber optically coupled image intensifier in the near future with its more efficient output phosphor, routine operation at the desigil value of $\Delta \mathrm{t}=5 \mathrm{msec}$ appears assured. As mentioned above, the detecior output exhibits a slight nonlinearity in its response to total incident light. Likewise, a small amount of nonlinearity ( $<10 \%$ ) is incroduced in the video disk archiving and re-digitizing. A simple look-up table transformation of the digitized data corrects for these effects in general.

An example of the raw data archived on the video disk is shown in Fig. 3. In addition to the two raw data images, the archived frame contains a linear intensity reference scale and labelling information. The data shown are examples of the detection of an internal disruption, or sawtooth crash, in a neutral beam-heated PBX-M plasma. The data field on the left was taken before the sawtooth collapse and the SXR emissions appear to be very intense and concentrated in the plasma center, while the second field on the right was taken after the sawtooth collapse and shows the emissions to be weaker and more spatially expanded, corresponding to the impurity expulsion from the plasma core and the flattening of the electron temperature profile. This particular discharge had a relatively small small hot core region, resulting in a very localized emission region in the plasma core ard hence relatively small images.

Since the observed images are chordally integrated along the tangentially viewing lines of sight, the measured inage must be inverted to derive the desired poloidal emissivity 
contours. Before formally inverting the data, the image is corrected for nonlinearities in the camera and ODR, adjusted for any possible transtrission variations in the optics across the image, and spatially filtered to reduce minor high frequency noise. We typically employ the inversion technique of Fourier mode modelling by minimization of the spectral radius, which was described in our earlier paper ${ }^{6}$ Briefly, the measured image is reconstructed by building a poloidal emissivity distribution via a superposition of cantesian Fourier components in two dimensions. This inversion is performed on a $31 \times 31$ pixel grid which is derived by subsampling the smoothed data. At present, the inversion calculations are done on the VAX computer with inrages uploaded from the local mictocomputer.

An example of the poloidal emission distribution derived from this diagnostic is shown in Fig. $\mathbf{4}$ for an elongated PBX-M discharge. This particular discharge was a welldeveloped large plasma whose cold exterior had a bean-shaped cross section with an indentation of $\sim 15 \%$. While almost circular at the plasma axis, the emissivity contours show an elongation of $\sim 1.5$ at a radius of $-14 \mathrm{~cm}$ and 1.7 at $\sim 21 \mathrm{~cm}$. The highly noncircular shape of the internal flux surfaces is thus very evident, although the overall internal shape appears more Dee-shaped than the characteristic kidney-bean shape of the outermost flux surfaces of PBX-M. The lack of an appearance of indentation on the outer contours is not significant at these inner radii for several reasons. First, the plasma electron temperature and hence the soft X-ray emissivity is very low in the plasma periphery, resulting in no measurements out there. Second, these initial inversions were done with data that do not include corrections for sparial inhomogeneities in the coupling optics, which may underestimate the emission from off-axis sightlines. Finally, the inversion code does not yet take into accoint the vignetting of the plasma image on the lower major radius side by the presence of a large magnetic field shaping coil.

\section{IV.Discussion}

A simple tangentially viewing SXR pinhole camera provides an effective diagnostic for internal shape measurements of non-circular toroidally symmetric plasmas. The camera system deployed on PBX-M employs a non-standard video camera to achieve time resolutions of $5 \mathrm{msec}$ or less, enough to resolve internal disruptions in the plasma core. The use of a real-time image processing system allows efficient acquisition and analysis of this data, while data archiving on a video optical disk recorder allows for easy storage and retrieval of large amounts of image data without burdening the central data acquisition 
computer system. The present system has already shown that the internal shape of PBX-M plasmas is more highly elongated than those of its predecessor device PBX. The success of these initial measurements allows the commencement of efforts to derive information on the intemal plasma current distribution through quantitative analysis of these images.

Further development of this system will revolve around refinements in the present design. Full automation of the data acquisition and archive is a short term goal, as is the installation of the CAMAC-based camera controller interface. Camera development will center on optimization of the SXR-to-visible converter, and the use of multiple filters to separate density and temperature inhomogeneities in the plasma. Laboratory measurements are in progress to better characterize the energy response and efficiency of the image converter. Further development and refinement of the image inversion code are also planned for the near future. Finally, significant re-designs of the camera head assembly are under study for the application of this diagnostic to the high radiation environments expected in the next generation of fusion devices.

The authors wish to thank M. Okabayashi and $\mathrm{K}$. Bol for their support and encouragement for this effort. The technical support of D. Cylinder, and useful discussions with E. Silver, K Hill, and S. Sesnic are gratefully acknowledged. This work was supported by the U.S. Dept. of Energy Contract No. DE-AC02-76-CHO-3073. 


\section{References}

1 J. B. Christiansen and J. B. Taylor, Nucl. Fusion 22, 111 (1982).

2 N. C. Luhman and W. A. Peebles, Rev. Sci. Instrum. 55, 279 (1984).

3 J. F. Carnacho and R. F. Granetz, Rev. Sci. Instrum. 57, 417 (1986).

4 P. Smeulders, Nucl. Fusion 26, 267 (1986).

5 A. J. Lieber and R. Snider, Rev. Sci. Instrum. 56, 821 (1985).

6 R. J. Fonck, M. Reusch, K.P. Jaehnig, and P. Roney, SPIE Vol. $691 \underline{\text { X-Ray }}$ Imaging II 111 (1986).

7 A. Holland, R. J. Fonck, and S. Sesnic, these proceedings.

8 J. Schivell, IEEE Trans. on Plasma Sci. PS-8, 226 (1980).

9 G. F. Knoll, Radiation Detection and Measurement, (J. Wiley \& Sons, New York, 1979) 260.

10R. J. Fonck, A. T. Ramsey, and R. V. Yelle, Appl. Optics 21, 2115 (1982). 


\section{Figure Caprions}

Fig. 1. Schematic of the soft $\mathrm{X}$-ray pinhole camera optical assembly.

Fig. 2. Schematic of the electronic components for the camera control and data acquisition systems.

Fig. 3. Sample of raw data frame archived on the optical disk recorder. Two time exposures, labels, and a linear reference scale (not shown) are included on each frame.

Fig. 4. Sample inverted poloidal emissivity distribution derived from the tangential SXR image of a highly elongated PBX-M discharge. The contoured intensity scale increases montonically towards the discharge center, and intemal machine hardware is schematically drawn in the coordinate frame. 
$88 \times 3126$

FILTER WHEEL

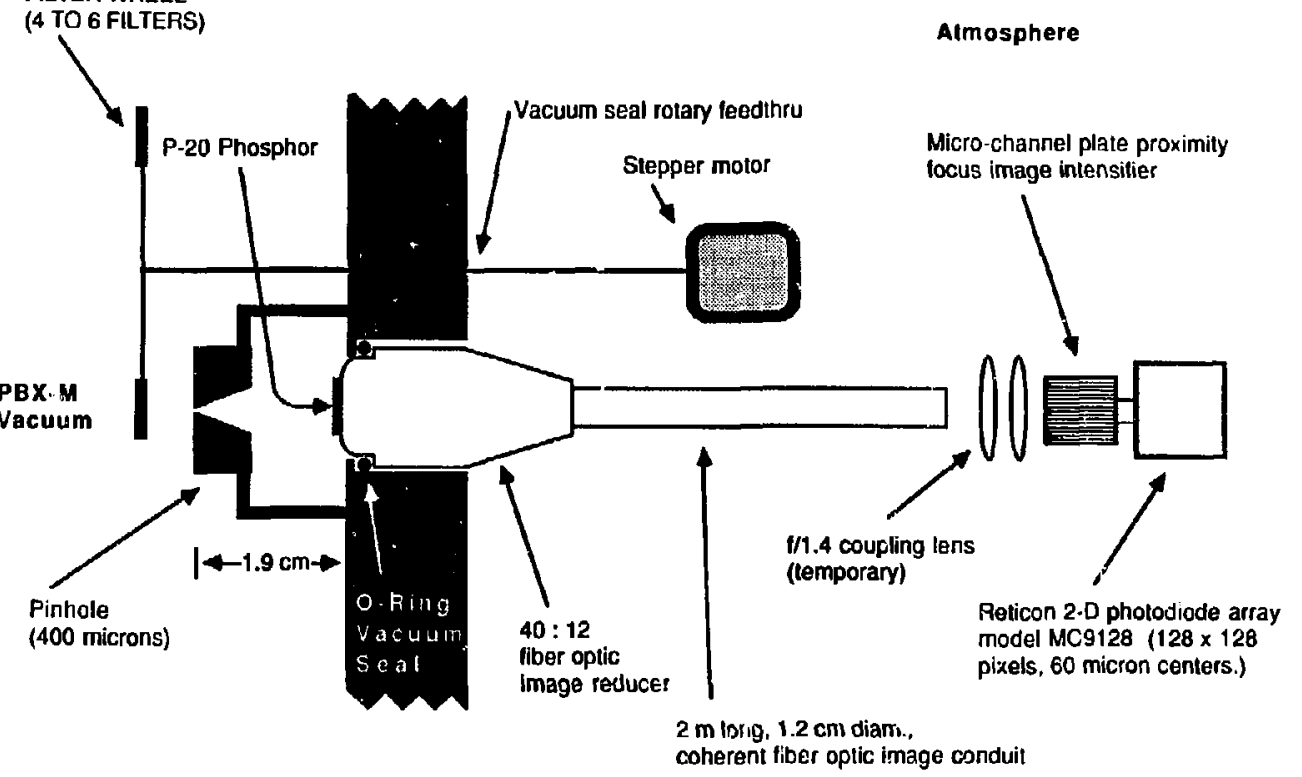

Figure 1 


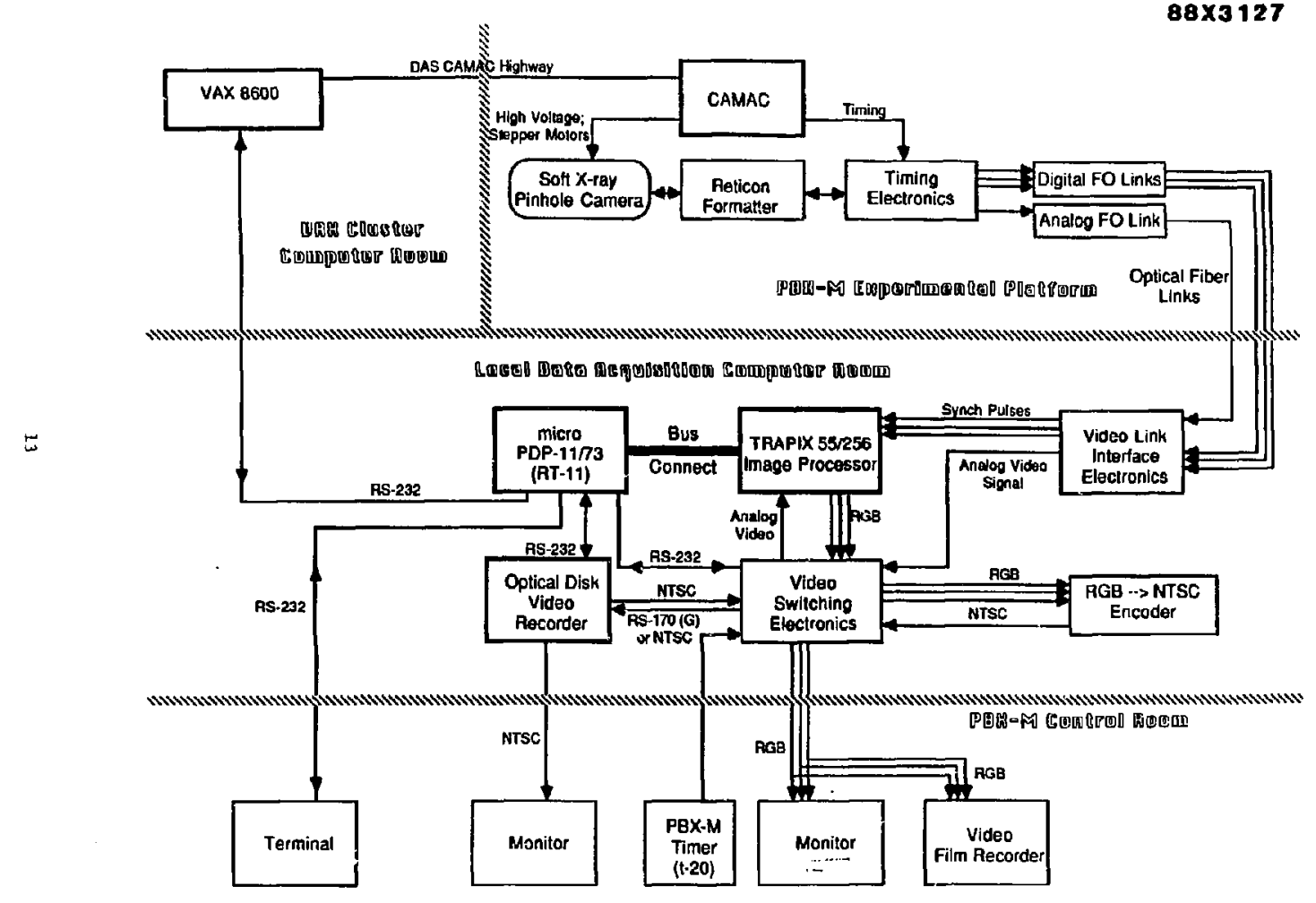


SHOT 227547? 15-1AR-86 17: 6:14 $\because \because:=: 9$ 3) $15:=8$

$501: 540 ; \quad, 510: 520$

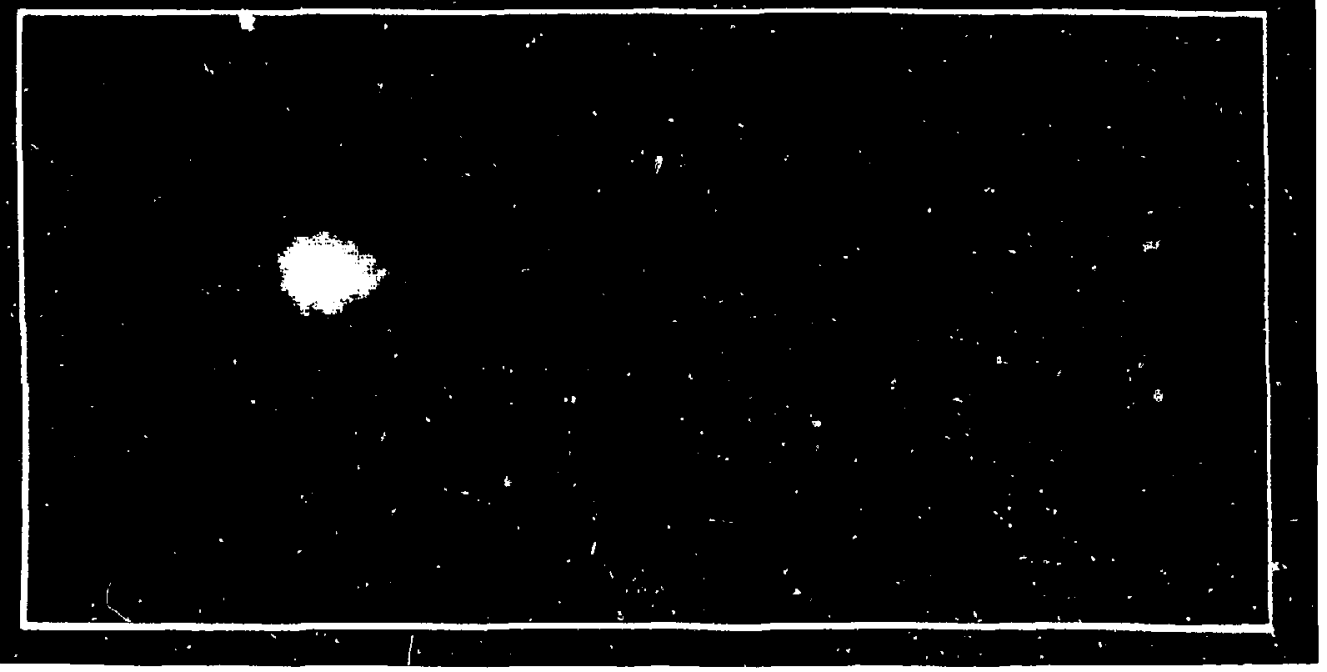

Figure 3 


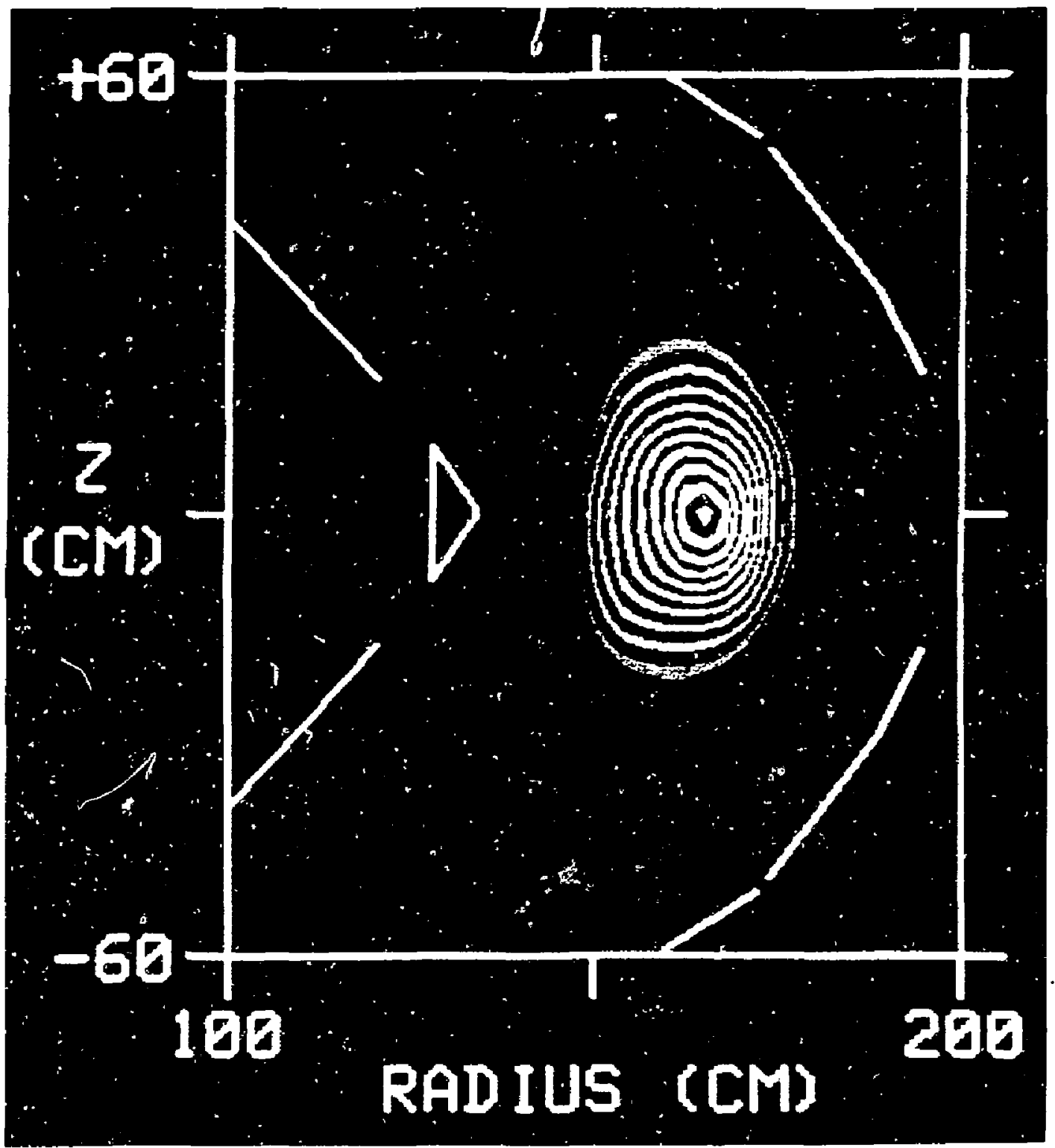

Flgure 4 